Chemistry of Heterocyclic Compounds 2017, 53(9), 935

\section{Rearrangements in the chemistry of heterocycles}

Molecular rearrangements are probably the most elegant type of organic chemistry reactions, comparable in their aesthetic appearance with the works of graphic artist M. C. Escher, who is most famous by his conceptual lithographs, metamorphoses, wood and metal carvings, and can be considered as the brightest representative of the "imp-art". The intramolecular rearrangement of ammonium cyanate to urea, generally known as the Wöhler reaction, laid the foundations of organic synthesis in 1828. It is difficult to convey the excitement of a modern synthetic chemist when a compound under certain conditions undergoes a rearrangement to a new product, while the molecular mass remains unchanged (intramolecular atomeconomical rearrangement). Equally great satisfaction is derived from attempts at deciphering the mechanisms of rearrangements and schematic representation of changes involving the movement of substituents, atoms, and electrons.

Molecular rearrangements are chemical reactions that result in changes of the connectivity between atoms in a molecule, the position of multiple bonds and their multiplicity, and which are accomplished with conservation of the atom composition of the molecule (isomerization) or involves changes of composition.

Molecular rearangements are not merely intellectually satisfying, but also serve as essential tools for synthetic chemists. A significant part of known rearrangements is relevant for the chemistry of heterocyclic compounds. Various types of rearrangements enable such transformations as ring expansion (Beckmann, Baeyer-Villiger, Meisenheimer, and Ciamician-Dennstedt rearrangements), ring contraction (Ramberg-Bäcklund, Mamedov, quasi-Favorskii rearrangements), transfer of functional groups (Payne, Mislow-Evans rearrangements). The chemistry of heterocycles includes rearrangements that do not affect the type of the heterocycle (Zincke, Dimroth rearrangements), replace the heteroatom with a different heteroatom (for example, recyclization of pyrilium salts to pyridines, thiopyrilium salts, or phosphobenzene derivatives), or even replace the heteroatom with a carbon atom (Hafner, Ferrier, Kost-Sagitullin rearrangements).

A synthetically important feature of many rearrangements is their stereospecificity. In many cases that can be used for determining the stereochemical characteristics of the starting compounds.

It should be pointed out that processes involving molecular rearangements have substantially affected the origin and development of a very valuable modern direction of organic synthesis, namely, green chemistry.

The classification of molecular rearrangements is possible according to various criteria. We could focus on the functional groups in the starting material or the type of product, or the

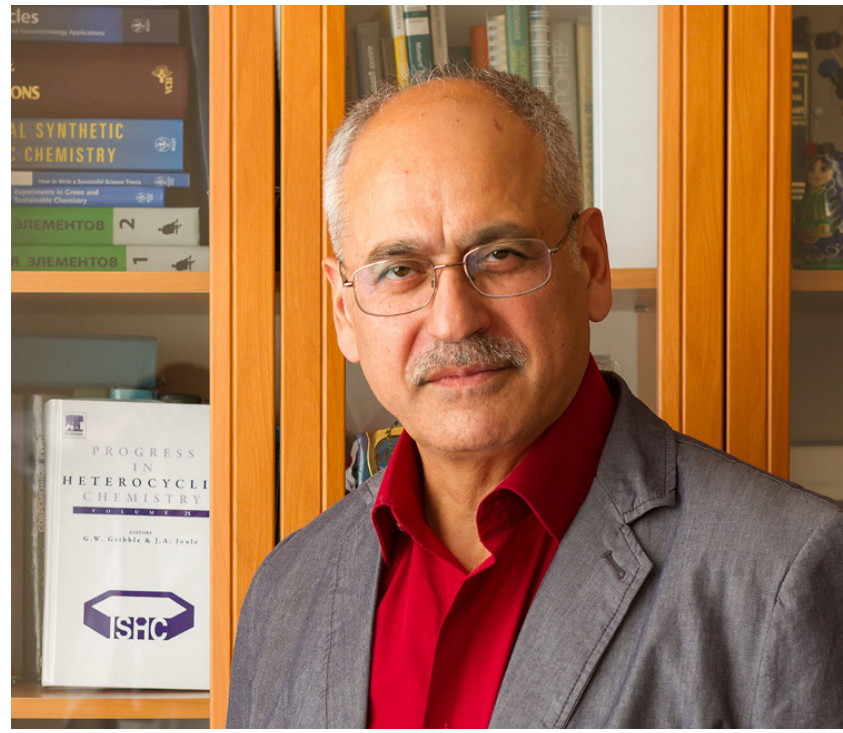

reaction mechanism. However, any classification will be incomplete to a certain degree. For example, from the point of view of reaction mechanism, classification may be difficult for some reactions, such as vinylcyclopropane-cyclopentene rearrangement or its heteroatom version, which can be a radical, ionic, or metal-catalyzed process.

This thematic issue was not intended for thorough analysis and classification of all known rearrangement reactions, which would be practically impossible. Our goal was to demonstrate advanced applications of known rearrangements and to present new reactions. The issue contains 5 reviews ( 1 in the part "Heterocycles in Focus"), 9 articles, and 3 brief communications.

I would like to express my gratitude to all authors who participated in the creation of this thematic issue, as well as the reviewers of manuscripts and members of the editorial team, who provided valuable comments and input for improving the quality of reviews and articles presented for your consideration. Hopefully, this thematic issue will be interesting and useful both for researchers in the field of synthetic organic chemistry, as well as specialists involved in medicinal and industrial chemistry, in the study of natural products, and the design of energetic materials.

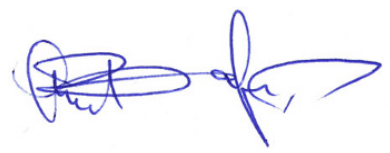

Editor of thematic issue Doctor of Chemical Sciences, Professor V. A. Mamedov, A. E. Arbuzov Institute of Organic and Physical Chemistry, Russian Academy of Sciences, Kazan, Russia 